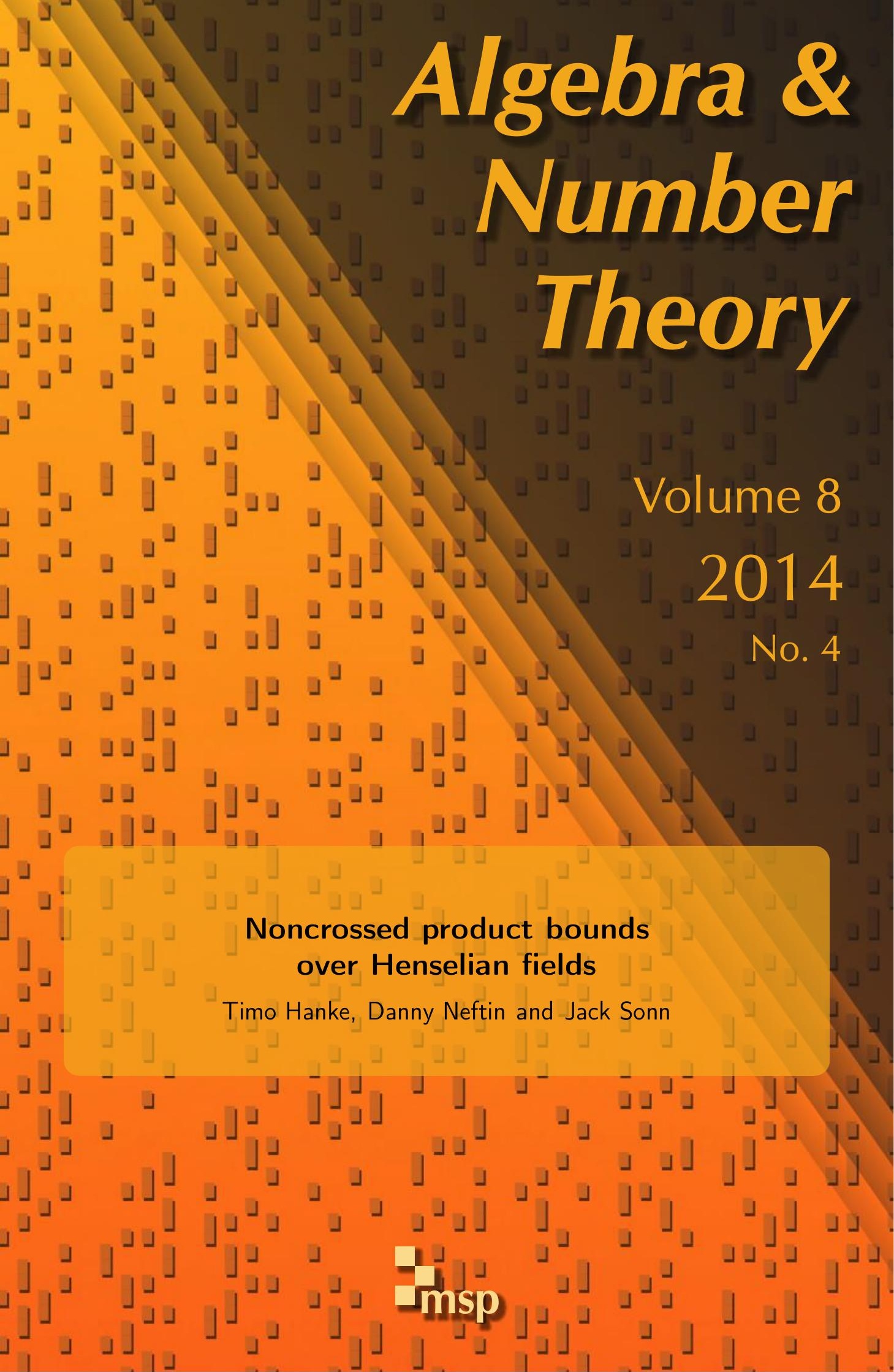




\title{
Noncrossed product bounds over Henselian fields
}

\author{
Timo Hanke, Danny Neftin and Jack Sonn
}

\begin{abstract}
The existence of noncrossed product division algebras (finite-dimensional central division algebras with no maximal subfield that is Galois over the center) was for a time the biggest open problem in the theory of division algebras, until it was settled by Amitsur.

Motivated by Brussel's discovery of noncrossed products over $\mathbb{Q}((t))$, we describe the "location" of noncrossed products in the Brauer group of general Henselian valued fields with arbitrary value group and global residue field. We show that within the fibers defined canonically by Witt's decomposition of the Brauer group of such fields, crossed products and noncrossed products are, roughly speaking, separated by an index bound. This generalizes a result of Hanke and Sonn for rank-1 valued Henselian fields.

Furthermore, we show that the new index bounds are of different nature from the rank-1 case. In particular, all fibers not covered by the rank-1 case contain noncrossed products, unless the residue characteristic interferes.
\end{abstract}

\section{Introduction}

A finite-dimensional division algebra over its center $F$ is called a crossed product if it has a maximal commutative subfield which is Galois over $F$, and otherwise a noncrossed product.

After Amitsur [1972] settled the fundamental long-standing problem of existence of noncrossed products, they were discovered over more familiar fields. Most notably, Brussel [1995; 2002] showed that noncrossed products exist over complete discrete rank-1 valued fields with a global residue field, ${ }^{1}$ for example, over $\mathbb{Q}((x))$. From this basic case, their existence over many other fields was derived, for example, over all finitely generated fields that are neither finite nor global [Brussel 2002], and over all function fields of curves over complete discrete valuation rings [Brussel 2001; Chen 2010].

MSC2010: primary 16S35; secondary 11R32, $12 \mathrm{~F} 12$.

Keywords: noncrossed product, division algebra, Henselian fields.

${ }^{1} \mathrm{By}$ a global field we mean a finite extension of $\mathbb{Q}$ or a finite extension of $\mathbb{F}_{q}(t)$, where $\mathbb{F}_{q}$ is a finite field. 
The basic setup used for Brussel's discovery is Witt's description [1936] of the Brauer group $\operatorname{Br}(F)$ of a complete discrete rank-1 valued field. More precisely, Witt's theorem describes the inertially split part $\operatorname{SBr}(F)$, which consists of all elements of $\operatorname{Br}(F)$ split by an unramified extension (see [Hanke 2011, §5]). Witt's theorem applies more generally to Henselian fields $F$ with arbitrary value group $\Gamma$ (see [Scharlau 1969; Jacob and Wadsworth 1990, §5; Aljadeff et al. 2007, Theorem 3.2]; or the section titled "The canonical Brauer group filtration" in [Tignol and Wadsworth $\geq 2014]$ ), for example, to the field of iterated Laurent series $\mathbb{Q}\left(\left(x_{1}\right)\right) \ldots\left(\left(x_{r}\right)\right)$, and gives an isomorphism

$$
\operatorname{SBr}(F) \cong \operatorname{Br}(K) \oplus \operatorname{Hom}_{c}\left(G_{K}, \Delta / \Gamma\right),
$$

where $K$ is the residue field and $G_{K}$ is its absolute Galois group equipped with the Krull topology, $\Delta$ is the divisible hull of $\Gamma, \Delta / \Gamma$ is equipped with the discrete topology, and $\mathrm{Hom}_{c}$ is the group of continuous homomorphisms.

Assume $K$ is a global field. Hanke and Sonn [2011] took the approach of fixing an element $\chi \in \operatorname{Hom}_{c}\left(G_{K}, \Delta / \Gamma\right)$ and considering the fiber $\operatorname{Br}(K)+\chi$. We call $\chi$ cyclic if its image $\operatorname{Im}(\chi)$ is cyclic. For cyclic $\chi$, [Hanke and Sonn 2011] shows that for every $N \in \mathbb{N}$ there are only two possible cases:

(I) all division algebras of index $N$ in the fiber over $\chi$ are crossed products;

(II) the fiber over $\chi$ contains infinitely many noncrossed products of index $N$.

Furthermore, and most importantly, there are bounds on the exponents in the prime decomposition of $N$ such that case (I) occurs "below the bounds" and case (II) "above". A precise formulation of this result is the special case of Theorem 1.1 below, in which $\chi$ is assumed to be cyclic. ${ }^{2}$

Little was known about the appearance of noncrossed products in the more complicated case of noncyclic $\chi$. In fact, there were only two examples [Coyette 2012; Hanke 2004] of noncyclic $\chi$, the fiber of which contains noncrossed products.

In the present paper, we show that the phenomenon discovered in [Hanke and Sonn 2011] for cyclic $\chi$ holds more generally for arbitrary $\chi$ (Theorem 1.1). Moreover, we show that "away from char $K$ " noncrossed products appear in every noncyclic fiber (Theorem 1.2).

Note that by [Jacob and Wadsworth 1990, Theorem 5.15(a)], the index of an element in the fiber over $\chi$ is always a multiple of $|\chi|:=|\operatorname{Im}(\chi)|$.

Theorem 1.1. There exists a collection of bounds $b_{p}=b_{p}(\chi) \in \mathbb{N} \cup\{\infty\}$, where $p$ runs through the rational primes, such that, for every natural number $m=\prod p^{n_{p}}$,

\footnotetext{
${ }^{2}$ In order to see that Theorem 1.1 for cyclic $\chi$ was proved in [Hanke and Sonn 2011] not only for rank-1 valued fields, one should replace [Hanke and Sonn 2011, Brussel's Lemma, p. 322] by [Hanke 2011, Corollary 5].
} 
(a) if $n_{p} \leq b_{p}(\chi)$ for all $p$, then all division algebras of index $m|\chi|$ in the fiber over $\chi$ are crossed products;

(b) if $n_{p}>b_{p}(\chi)$ for some $p$, then the fiber over $\chi$ contains infinitely many noncrossed products of index $m|\chi|$.

Our proof of Theorem 1.1 includes the case of cyclic $\chi$ and is simpler than [Hanke and Sonn 2011].

Note that $b_{p}(\chi)=\infty$ is allowed, and hence, as shown in [Hanke and Sonn 2011], it may happen that for some cyclic $\chi$, only (I) occurs. However, in striking contrast to the cyclic case, we show:

Let $M$ be the fixed field of the kernel of $\chi$.

Theorem 1.2. If $p \neq \operatorname{char} K$ and the $p$-Sylow subgroup of $\operatorname{Im}(\chi)$ is noncyclic, then:

(i) $b_{p}(\chi)<\infty$;

(ii) if $M$ does not contain the $p$-th roots of unity, then $b_{p}(\chi)=0$.

In particular, the fiber over $\chi$ contains noncrossed products whenever the maximal prime-to-char $K$ subgroup of $\operatorname{Im}(\chi)$ is noncyclic.

This is in contrast to the cyclic case because, according to [Hanke and Sonn 2011], $b_{p}(\chi)=\infty$ can occur for cyclic $\chi$, even if $M$ does not contain the $p$-th roots of unity. Thus, neither of statements (i) and (ii) of Theorem 1.2 holds for cyclic $\chi$.

Section 4 demonstrates, for noncyclic $\chi$, how a description of the bounds obtained from the proof of Theorem 1.1 can be used to compute the bounds in examples. In particular, we obtain new noncrossed products of low index (Examples 4.1, 4.3) and show that, unlike in the rank- 1 case, the value of the bound $b_{p}(\chi)$ can be zero regardless of the number of roots of unity in $M$.

\section{Existence of bounds}

Setup. Let $F$ be a Henselian valued field with value group $\Gamma$ and residue field a global field $K$. Let $\Delta$ denote the divisible hull of $\Gamma$. We fix an isomorphism as in (1-1) and write $\alpha+\chi$ to denote an element of $\operatorname{SBr}(F)$ corresponding to $(\alpha, \chi)$.

Throughout the paper, we consider the character $\chi \in \operatorname{Hom}_{c}\left(G_{K}, \Delta / \Gamma\right)$ as fixed. We let $M$ denote the fixed field of the kernel of $\chi$, which is a finite abelian extension of $K$ with Galois group $\operatorname{Im}(\chi)$.

Let $\mathbb{P}$ be the set of finite rational primes, and for any $p \in \mathbb{P}, n \in \mathbb{N}$, denote by $v_{p}(n)$ the maximal exponent $e$ such that $p^{e} \mid n$.

Towards the proof of Theorem 1.1. For $\alpha \in \operatorname{Br}(K)$, we denote by $\alpha^{M}$ the image of $\alpha$ in $\operatorname{Br}(M)$ under the restriction map. The index formula [Jacob and Wadsworth 
1990, Theorem 5.15(a) $]^{3}$ gives

$$
\operatorname{ind}(\alpha+\chi)=|\chi| \text { ind } \alpha^{M} .
$$

Therefore, in order to prove Theorem 1.1, we consider the following condition on $\chi$ :

For every $\alpha \in \operatorname{Br}(K)$ with ind $\alpha^{M}=m$, the division algebra underlying $\alpha+\chi$ is a crossed product.

For a global field $K$, we shall prove the existence of bounds $b_{p}(\chi)$ such that $\left(\mathrm{I}_{m}\right)$ holds if and only if $v_{p}(m) \leq b_{p}(\chi)$ for all $p \in \mathbb{P}$. The details of our proof will reveal that if $\left(\mathrm{I}_{m}\right)$ does not hold, then there are in fact infinitely many $\alpha \in \operatorname{Br}(K)$ with ind $\alpha^{M}=m$ such that the division algebra underlying $\alpha+\chi$ is a noncrossed product (Remark 2.9). The proof of Theorem 1.1 will then be completed.

Galois covers. We say that a field $L \supseteq M$ is a cover of $M / K$ if $L / K$ is Galois. In this case, we call $m:=[L: M]$ the degree of the cover and speak of $L$ as an $m$-cover.

The division algebra underlying $\alpha+\chi$ is a crossed product if and only if the division algebra underlying $\alpha^{M}$ contains a maximal subfield which is Galois over $K$ (see [Hanke 2011, Corollary 5; Brussel 1995, p. 381, Corollary] for complete discrete rank-1 valued $F$ ). Such maximal subfields are characterized as the $m$ covers of $M / K$ that split $\alpha$, where $m=$ ind $\alpha^{M}$ (see, for example, [Pierce 1982, Corollary 13.3]). Condition $\left(\mathrm{I}_{m}\right)$ is therefore equivalent to:

Every $\alpha \in \operatorname{Br}(K)$ with ind $\alpha^{M}=m$ is split by an $m$-cover of $M / K$.

$\left(\mathrm{A}_{m}\right)$

Remark 2.1. (i) For the equivalence of $\left(\mathrm{I}_{m}\right)$ and $\left(\mathrm{A}_{m}\right)$ it is not required that $K$ be a global field.

(ii) Condition $\left(\mathrm{A}_{m}\right)$ is a condition on $M$ rather than on $\chi \cdot\left(\mathrm{A}_{m}\right)$ can be considered more generally for any Galois extension $M / K$. In fact, from now on $M$ may be replaced by an arbitrary finite Galois extension of the global field $K$.

Local and global splitting covers. Let $L$ be a cover of the fixed Galois extension $M / K$. We write $K_{\mathfrak{p}}$ for the completion at $\mathfrak{p}$ and $[L: M]_{\mathfrak{p}}:=\left[L_{\mathfrak{P}}: M_{\mathfrak{P} \cap M}\right]$ for the local degree of $L$ at $\mathfrak{p}$, where $\mathfrak{P}$ is any prime of $L$ dividing $\mathfrak{p}$.

Let $\alpha \in \operatorname{Br}(K)$. For a prime $\mathfrak{p}$ of $K$, let $\operatorname{ind}_{\mathfrak{p}} \alpha:=\operatorname{ind}\left(\alpha^{K_{\mathfrak{p}}}\right)$ and $\operatorname{inv}_{\mathfrak{p}} \alpha \in \mathbb{Q} / \mathbb{Z}$ be the Hasse invariant at $\mathfrak{p}$. Recall (see, for example, [Pierce 1982, §17.10]) that $\operatorname{inv}_{\mathfrak{p}} \alpha$ is of order $\operatorname{ind}_{\mathfrak{p}} \alpha$ and that $\operatorname{inv}_{\mathfrak{P}} \alpha^{L}=[L: K]_{\mathfrak{p}} \operatorname{inv}_{\mathfrak{p}} \alpha$ for any Galois extension $L / K$ and $\mathfrak{P} \mid \mathfrak{p}$. Thus, $L_{\mathfrak{P}}$ splits $\alpha^{K_{\mathfrak{p}}}$ if and only if $\operatorname{ind}_{\mathfrak{p}} \alpha \mid[L: K]_{\mathfrak{p}}$, and $L_{\mathfrak{P}}$ embeds into the division algebra underlying $\alpha^{K_{\mathfrak{p}}}$ if and only if $[L: K]_{\mathfrak{p}} \mid \operatorname{ind}_{\mathfrak{p}} \alpha$. We also get

$$
v_{p}\left(\operatorname{ind}_{\mathfrak{p}} \alpha\right) \leq v_{p}\left(\operatorname{ind}_{\mathfrak{p}} \alpha^{L}\right)+v_{p}\left([L: K]_{\mathfrak{p}}\right),
$$

3 Theorem 5.15(a) of [Jacob and Wadsworth 1990] does not require that $K$ be a global field. 
where $\operatorname{ind}_{\mathfrak{p}} \alpha^{L}:=\operatorname{ind} \alpha^{L_{\mathfrak{P}}}$ for any prime $\mathfrak{P}$ of $L$ dividing $\mathfrak{p}$. Furthermore, (2-1) is an equality if $v_{p}\left(\operatorname{ind}_{\mathfrak{p}} \alpha^{L}\right)>0$.

By the theorem of Albert, Brauer, Hasse, and Noether (see, for example, [Pierce $1982, \S 18.4]$ ), a Brauer class over a global field is split by a field $L$ if and only if its completions are split by $L$. In particular, for any cover $L$ of $M / K$,

$L$ splits $\alpha$ if and only if $\operatorname{ind}_{\mathfrak{p}} \alpha^{M} \mid[L: M]_{\mathfrak{p}}$ for all primes $\mathfrak{p}$ of $K$.

Limits on local indices. Our first goal is to translate $\left(\mathrm{A}_{m}\right)$ to a condition about the existence of $M / K$ with prescribed local degrees at finitely many primes of $K$ (see Proposition 2.8 below). For this, in view of (2-2), we analyze the possible local indices $\operatorname{ind}_{\mathfrak{p}} \alpha^{M}$. We describe upper limits for the possible local indices in Lemmas 2.3 and 2.4, using the following terminology:

Definition 2.2. For $p \in \mathbb{P}$, let $u_{p}^{(1)}, u_{p}^{(2)}, \ldots$ be the family of numbers $v_{p}\left([M: K]_{\mathfrak{p}}\right)$, where $\mathfrak{p}$ runs over the primes of $K$, ordered so that $u_{p}^{(1)} \geq u_{p}^{(2)} \geq \ldots$ If $u_{p}^{(1)}>u_{p}^{(2)}$, then the unique prime $\mathfrak{p}$ of $K$ with $v_{p}\left([M: K]_{\mathfrak{p}}\right)=u_{p}^{(1)}$ is called $p$-isolated in $M / K$. We denote by $g_{p}$ the gap $u_{p}^{(1)}-u_{p}^{(2)}$, so that $g_{p}>0$ if and only if there is a $p$-isolated prime. We shall call a prime $\mathfrak{p}$ of $K$ isolated if it is $p$-isolated for some $p \in \mathbb{P}$.

Let $U_{p}$ be the set of primes $\mathfrak{p}$ of $K$ for which $v_{p}\left([M: K]_{\mathfrak{p}}\right) \geq u_{p}^{(2)}$. Let $U$ consist of the isolated primes in $M / K$, and if $\left|U_{2}\right|$ is finite and odd, also of the primes in $U_{2}$.

The following properties are deduced from Chebotarev's density theorem. For every infinite prime $\mathfrak{q}$ of $K,[M: K]_{\mathfrak{q}}$ is at most 2 . If $2 \mid[M: K]$, by Chebotarev's theorem, there is a finite prime $\mathfrak{p}$ such that $[M: K]_{\mathfrak{p}}=2$. In particular, infinite primes are nonisolated, and for every $i \in \mathbb{N}$ there is a finite prime $\mathfrak{p}_{i}$ with

$$
v_{p}\left([M: K]_{\mathfrak{p}_{i}}\right)=u_{p}^{(i)} .
$$

Moreover, for any prime $\mathfrak{q}$ of $K$ unramified in $M$, by Chebotarev's theorem, there is a prime $\mathfrak{p}$ with $[M: K]_{\mathfrak{p}}=[M: K]_{\mathfrak{q}}$. Hence, isolated primes must ramify nontrivially in $M / K$, and the set $U$ is finite. For every $p \in U_{p}$, the set $U_{p}$ can be infinite, and in view of (2-3), it contains at least two finite primes.

For $m \in \mathbb{N}$ and a prime $\mathfrak{p}$ of $K$, define

$$
w_{p}(m, \mathfrak{p}):= \begin{cases}v_{p}(m) & \text { if } \mathfrak{p} \text { is non- } p \text {-isolated } \\ \max \left\{v_{p}(m)-g_{p}, 0\right\} & \text { if } \mathfrak{p} \text { is } p \text {-isolated. }\end{cases}
$$

Note that the dependence on $\mathfrak{p}$ is marginal ${ }^{4}$ and that clearly $w_{p}(m, \mathfrak{p}) \leq v_{p}(m)$.

${ }^{4} \mathrm{~A}$ reader who for the time being decides to disregard the possible appearance of isolated primes may substitute $v_{p}(m)$ for $w_{p}(m, \mathfrak{p})$. 
Lemma 2.3. For every $\alpha \in \operatorname{Br}(K)$ and prime $\mathfrak{p}$ of $K$,

$$
v_{p}\left(\operatorname{ind}_{\mathfrak{p}} \alpha^{M}\right) \leq w_{p}\left(\text { ind } \alpha^{M}, \mathfrak{p}\right) .
$$

Proof. Set $k:=v_{p}\left(\operatorname{ind}_{\mathfrak{p}} \alpha^{M}\right), n:=v_{p}\left(\right.$ ind $\left.\alpha^{M}\right)$, and let $u_{p}^{(1)}, u_{p}^{(2)}$ be as above. For a non- $p$-isolated prime $\mathfrak{p}$, the assertion holds since $k \leq n$. Assume that $\mathfrak{p}$ is $p$-isolated. The assertion to prove is: $k=0$ or $n \geq k+g_{p}$. Assume $k>0$. By (2-1),

$$
v_{p}\left(\operatorname{ind}_{\mathfrak{p}} \alpha\right)=v_{p}\left(\operatorname{ind}_{\mathfrak{p}} \alpha^{M}\right)+v_{p}\left([M: K]_{\mathfrak{p}}\right)=k+u_{p}^{(1)} .
$$

Since the sum of Hasse invariants of $\alpha$ is 0 , there exists a prime $\mathfrak{p}_{1} \neq \mathfrak{p}$ of $K$ with $v_{p}\left(\operatorname{ind}_{\mathfrak{p}_{1}} \alpha\right) \geq k+u_{p}^{(1)}$. Since $v_{p}\left([M: K]_{\mathfrak{p}_{1}}\right) \leq u_{p}^{(2)},(2-1)$ gives

$$
n \geq v_{p}\left(\operatorname{ind}_{\mathfrak{p}_{1}} \alpha^{M}\right) \geq v_{p}\left(\operatorname{ind}_{\mathfrak{p}_{1}} \alpha\right)-v_{p}\left([M: K]_{\mathfrak{p}_{1}}\right) \geq k+u_{p}^{(1)}-u_{p}^{(2)}=k+g_{p} .
$$

To prove the second restriction, we use the following inequality, which holds ${ }^{5}$ for every prime $\mathfrak{p}$ of $K, p \in \mathbb{P}$, and $m \in \mathbb{N}$ :

$$
w_{p}(m, \mathfrak{p})+v_{p}\left([M: K]_{\mathfrak{p}}\right) \leq v_{p}(m)+u_{p}^{(2)},
$$

with equality if and only if $\mathfrak{p} \in U_{p}$.

Lemma 2.4. Let $\alpha \in \operatorname{Br}(K)$ with $v_{2}\left(\right.$ ind $\left.\alpha^{M}\right)>g_{2}$ and let $p=2$. Then the number of primes $\mathfrak{p} \in U_{2}$ for which (2-5) is an equality is even.

Proof. Let $m=$ ind $\alpha^{M}$. Let $U_{2, \alpha}$ be the set of primes $\mathfrak{p} \in U_{2}$ for which (2-5) is an equality. Then (2-1), (2-5), and (2-6) imply

$$
\begin{aligned}
v_{2}\left(\operatorname{ind}_{\mathfrak{p}} \alpha\right) & \leq v_{2}\left(\operatorname{ind}_{\mathfrak{p}} \alpha^{M}\right)+v_{2}\left([M: K]_{\mathfrak{p}}\right) \\
& \leq w_{2}\left(\text { ind } \alpha^{M}, \mathfrak{p}\right)+v_{2}\left([M: K]_{\mathfrak{p}}\right) \leq v_{2}\left(\text { ind } \alpha^{M}\right)+u_{2}^{(2)}
\end{aligned}
$$

for every prime $\mathfrak{p}$ of $K$, with equalities for every $\mathfrak{p} \in U_{2, \alpha}$. Since (2-6) is strict for $\mathfrak{p} \notin U_{2}$ and (2-5) is strict for $\mathfrak{p} \in U_{2} \backslash U_{2, \alpha},(2-7)$ is strict for every prime $\mathfrak{p} \notin U_{2, \alpha}$.

Let $r:=v_{2}\left(\right.$ ind $\left.\alpha^{M}\right)+u_{2}^{(2)}$. Since (2-7) holds for all $\mathfrak{p}$, we have $v_{2}($ ind $\alpha) \leq r$ with equality if and only if $U_{2, \alpha}$ is nonempty. If $v_{2}$ (ind $\left.\alpha\right)<r$, then $U_{2, \alpha}$ is empty and the assertion holds. Thus, we may assume $v_{2}($ ind $\alpha)=r$ and write ind $\alpha=2^{r} m^{\prime}$ for odd $m^{\prime}$. Then the class $\alpha^{\prime}:=\alpha^{2^{r-1} m^{\prime}} \in \operatorname{Br}(K)$ has exponent 2, nontrivial Hasse invariants at primes of $U_{2, \alpha}$, and trivial invariants outside $U_{2, \alpha}$. Since the sum of invariants of $\alpha^{\prime}$ is $0,\left|U_{2, \alpha}\right|$ is even.

Lemmas 2.3 and 2.4 lead to upper limits on local indices (see Proposition 2.8). We shall now construct elements which attain these upper bounds. We divide the construction into several cases:

${ }^{5}$ This inequality is easily verified separately for $p$-isolated and non- $p$-isolated primes. 
Definition 2.5. For a finite set $S$ of primes of $K$ and $m \in \mathbb{N},(S, m)$ is balanced if $v_{2}(m) \leq g_{2}$ or $\left|S \cap U_{2}\right|$ is even. We say that $(S, m)$ is balanceable if $S$ can be enlarged so that $(S, m)$ is balanced, and otherwise unbalanceable.

Remark 2.6. Note that $(S, m)$ is unbalanceable if and only if $\left|U_{2}\right|$ is odd, $S \supseteq U_{2}$, and $v_{2}(m)>g_{2}$. In particular, $(S, m)$ can be unbalanceable only if $m$ is even.

Lemma 2.4 shows that if $\left(S, 2^{n}\right)$ is unbalanceable, then there are no elements $\alpha \in \operatorname{Br}(K)$ with ind $\alpha^{M}=2^{n}$, and $\operatorname{ind}_{\mathfrak{p}} \alpha^{M}=2^{w_{2}\left(2^{n}, \mathfrak{p}\right)}$ for every $\mathfrak{p} \in S$. The following lemma constructs such elements if $(S, m)$ is balanced.

Lemma 2.7. Let $n$ be a positive integer, $p \in \mathbb{P}, m=p^{n}$, and $\mathfrak{q} \in U_{2}$. Let $S$ be a finite set of primes of $K$ which contains at least two finite primes of $U_{p}$.

Then there exists $\alpha \in \operatorname{Br}(K)$, with ind $\alpha^{M}=m$, $\operatorname{ind}_{\mathfrak{p}} \alpha^{M}=1$ for all $\mathfrak{p} \notin S$ and $\operatorname{ind}_{\mathfrak{p}} \alpha^{M}=\operatorname{gcd}(p, 2)$ for every real $\mathfrak{p} \in S$ which is unramified in $M$, such that

(i) if $(S, m)$ is balanced, then $\operatorname{ind}_{\mathfrak{p}} \alpha^{M}=p^{w_{p}(m, \mathfrak{p})}$ for all finite $\mathfrak{p} \in S$;

(ii) if $(S, m)$ is unbalanceable, then $p=2, \mathfrak{q} \in S, w_{2}(m, \mathfrak{q})>0, \operatorname{ind}_{\mathfrak{p}} \alpha^{M}=2^{w_{2}(m, \mathfrak{p})}$ for all finite $\mathfrak{p} \in S \backslash\{\mathfrak{q}\}$, and $2^{w_{2}(m, \mathfrak{q})-1} \mid \operatorname{ind}_{\mathfrak{q}} \alpha^{M}$.

Proof. Write $S_{p}:=U_{p} \cap S$. Note that if $(S, m)$ is unbalanceable then $p=2, U_{2} \subseteq S$, and $n-g_{2}>0$, and hence $\mathfrak{q} \in U_{2} \subseteq S$ and $w_{2}(m, \mathfrak{q}) \geq n-g_{2}>0$.

If $p=2$ and $(S, m)$ is balanced, we claim that $\left|S_{2}\right|$ can be assumed to be even. Indeed, if $\left|S_{2}\right|$ is odd, then $n \leq g_{2}, M / K$ has a 2-isolated prime $\mathfrak{p}_{1}$, and $w_{2}\left(m, \mathfrak{p}_{1}\right)=0$. Hence by Lemma 2.3 , for any $\alpha \in \operatorname{Br}(K)$ with ind $\alpha^{M}=m$, one has $\operatorname{ind}_{\mathfrak{p}_{1}} \alpha^{M}=1$. Thus, we may add or remove $\mathfrak{p}_{1}$ from $S$ without changing the desired assertion. We may therefore assume that $\left|S_{2}\right|$ is even, proving the claim.

We define $\alpha$ by setting its Hasse invariants. Let $\mathfrak{q}_{1}, \mathfrak{q}_{2}$ be two distinct finite primes in $S_{p}$. If $(S, m)$ is unbalanceable, assume $\mathfrak{q}_{1}=\mathfrak{q}$. Set $\operatorname{inv}_{\mathfrak{p}} \alpha$ to be of order $p^{r_{\mathfrak{p}}}$, where $r_{\mathfrak{p}}=n+v_{p}\left([M: K]_{\mathfrak{p}}\right)$ for every finite $\mathfrak{p} \in S \backslash S_{p}$ and $r_{\mathfrak{p}}=n+u_{p}^{(2)}$ for every finite $\mathfrak{p} \in S_{p} \backslash\left\{\mathfrak{q}_{1}, \mathfrak{q}_{2}\right\}$, and of order $\operatorname{gcd}(p, 2)$ for every real $\mathfrak{p} \in S \backslash\left\{\mathfrak{q}_{1}, \mathfrak{q}_{2}\right\}$.

The order of all invariants we have set so far divides $p^{n+u_{p}^{(2)}}$. If $(S, m)$ is balanced, we can set $\operatorname{inv}_{\mathfrak{q}_{2}} \alpha$ to be of order $p^{n+u_{p}^{(2)}}$ such that $x:=\sum_{\mathfrak{p} \in S \backslash\left\{\mathfrak{q}_{1}\right\}} \operatorname{inv}_{\mathfrak{p}} \alpha$ has order $p^{n+u_{p}^{(2)}}$. Note that this is possible for $p=2$ since the invariants which were set to be of order $2^{n+u_{2}^{(2)}}$ are at primes of $S_{2} \backslash\left\{\mathfrak{q}_{1}, \mathfrak{q}_{2}\right\}$, a set of even order. If $(S, m)$ is unbalanceable then $p=2, n+u_{2}^{(2)} \geq 2$, and we can set $\operatorname{inv}_{\mathfrak{q}_{2}} \alpha=a / 2^{n+u_{2}^{(2)}}$ for odd $a$ such that $x:=\sum_{\mathfrak{p} \in S \backslash\left\{\mathfrak{q}_{1}\right\}} \operatorname{inv}_{\mathfrak{p}} \alpha=b / 2^{n+u_{2}^{(2)}}$ with $b \not \equiv 0(\bmod 4)$. Note that this is possible since $a$ can be chosen to be congruent to either 1 or $3(\bmod 4)$.

Setting $\operatorname{inv}_{\mathfrak{q}_{1}} \alpha:=-x$ and $\operatorname{inv}_{\mathfrak{p}} \alpha=0$ for $\mathfrak{p} \notin S$ completes the definition of $\alpha$. By (2-1), we have

$$
v_{p}\left(\operatorname{ind}_{\mathfrak{p}} \alpha^{M}\right)=\max \left\{v_{p}\left(\operatorname{ind}_{\mathfrak{p}} \alpha\right)-v_{p}\left([M: K]_{\mathfrak{p}}\right), 0\right\} .
$$


For finite $\mathfrak{p} \in S \backslash S_{p}$, we have $v_{p}\left(\operatorname{ind}_{\mathfrak{p}} \alpha\right)=n+v_{p}\left([M: K]_{\mathfrak{p}}\right)$, and hence $\operatorname{ind}_{\mathfrak{p}} \alpha^{M}=p^{n}$ by (2-8). For finite $\mathfrak{p} \in S_{p} \backslash\left\{\mathfrak{q}_{1}\right\}$, we have $v_{p}\left(\operatorname{ind}_{\mathfrak{p}} \alpha\right)=n+u_{p}^{(2)}$, and hence (2-8) gives $\operatorname{ind}_{\mathfrak{p}} \alpha^{M}=p^{w_{p}(m, \mathfrak{p})}$ for all finite $\mathfrak{p} \in S \backslash\left\{\mathfrak{q}_{1}\right\}$, and $\operatorname{ind}_{\mathfrak{p}} \alpha^{M}=\operatorname{gcd}(p, 2)$ for all real $\mathfrak{p} \in S \backslash\left\{\mathfrak{q}_{1}\right\}$ unramified in $M / K$. If $\left(S, p^{n}\right)$ is balanced, $\operatorname{ind}_{\mathfrak{q}_{1}} \alpha=p^{n+u_{p}^{(2)}}$, and hence by (2-8) we have $\operatorname{ind}_{\mathfrak{q}_{1}} \alpha^{M}=p^{w_{p}\left(p^{n}, \mathfrak{q}_{1}\right)}$, as required.

If $\left(S, p^{n}\right)$ is unbalanceable then $p=2,2^{n+u_{2}^{(2)}-1} \mid \operatorname{ind}_{\mathfrak{q}_{1}} \alpha, n>g_{2}$, and hence $w_{2}\left(2^{n}, \mathfrak{q}_{1}\right)>0$. Thus, $(2-8)$ and (2-6) give

$$
v_{2}\left(\operatorname{ind}_{\mathfrak{q}_{1}} \alpha^{M}\right) \geq n+u_{2}^{(2)}-1-v_{2}\left([M: K]_{\mathfrak{q}_{1}}\right)=w_{2}\left(2^{n}, \mathfrak{q}_{1}\right)-1 .
$$

Since $\operatorname{ind}_{\mathfrak{p}} \alpha^{M} \mid p^{n}$ for all $\mathfrak{p}$, ind $\alpha^{M} \mid p^{n}$. Since $S_{p}$ contains two finite primes, at least one of these primes $\mathfrak{p}$ satisfies $w_{p}(m, \mathfrak{p})=n$, and hence ind $\alpha^{M}=$ ind $\alpha_{\mathfrak{p}}^{M}=p^{n}$.

Covers with prescribed local degrees. We can now translate $\left(\mathrm{A}_{m}\right)$ to a condition on the local degrees of covers. Let $m \in \mathbb{N}$. For a finite prime $\mathfrak{p}$ of $K$, define $d_{\mathfrak{p}}(m) \in \mathbb{N}$ by requiring $p \in \mathbb{P}$ for every $v_{p}\left(d_{\mathfrak{p}}(m)\right)=w_{p}(m, \mathfrak{p})$. For an infinite prime $\mathfrak{p}$ of $K$, set $d_{\mathfrak{p}}(m):=\operatorname{gcd}(m, 2)$ if $\mathfrak{p}$ is real and unramified in $M$ and $d_{\mathfrak{p}}(m):=1$ otherwise.

Clearly, $d_{\mathfrak{p}}(m) \mid m$ for any $\mathfrak{p}$, and $d_{\mathfrak{p}}(m)=m$ if $\mathfrak{p}$ is finite and nonisolated.

Proposition 2.8. Condition $\left(\mathrm{A}_{m}\right)$ is equivalent to:

For every finite set $S$ and $\mathfrak{q} \in U_{2}, M / K$ has an $m$-cover $L$ such that $d_{\mathfrak{p}}(m) \mid[L: M]_{\mathfrak{p}}$ for every $\mathfrak{p} \in S$, except for $\mathfrak{p}=\mathfrak{q}$ when $(S, m)$ is unbalanceable, in which case $\left(d_{\mathfrak{q}}(m) / 2\right) \mid[L: M]_{\mathfrak{q}}$.

Proof. $\left(\mathrm{B}_{m}\right) \Longrightarrow\left(\mathrm{A}_{m}\right)$ : Let $\alpha \in \operatorname{Br}(K)$ with ind $\alpha^{M}=m$. Let $S$ be the set of primes $\mathfrak{p}$ of $K$ such that $\operatorname{ind}_{\mathfrak{p}} \alpha \neq 1$. For every finite $\mathfrak{p}$ and $p \in \mathbb{P}$, Lemma 2.3 implies

$$
v_{p}\left(\operatorname{ind}_{\mathfrak{p}} \alpha^{M}\right) \leq w_{p}(m, \mathfrak{p})=v_{p}\left(d_{\mathfrak{p}}(m)\right) .
$$

If $\mathfrak{p}$ is real and unramified in $M, \operatorname{ind}_{\mathfrak{p}} \alpha^{M} \mid \operatorname{gcd}(m, 2)=d_{\mathfrak{p}}(m)$. Thus, $\operatorname{ind}_{\mathfrak{p}} \alpha \mid d_{\mathfrak{p}}(m)$ for all primes $\mathfrak{p}$ of $K$. If $(S, m)$ is balanceable, $\left(\mathrm{B}_{m}\right)$ gives an $m$-cover $L$ for which $\operatorname{ind}_{\mathfrak{p}} \alpha^{M} \mid[L: M]_{\mathfrak{p}}$ for all $\mathfrak{p} \in S$. Hence, by (2-2), $L$ splits $\alpha$.

Assume $(S, m)$ is unbalanceable. By Lemma 2.4, there is a prime $\mathfrak{q} \in U_{2} \subseteq S$ for which

$$
v_{2}\left(\operatorname{ind}_{\mathfrak{q}} \alpha^{M}\right) \leq w_{2}(m, \mathfrak{q})-1 .
$$

It follows that $\operatorname{ind}_{\mathfrak{p}} \alpha^{M} \mid d_{\mathfrak{p}}(m)$ for all $\mathfrak{p} \neq \mathfrak{q}$ and $\operatorname{ind}_{\mathfrak{q}} \alpha^{M} \mid\left(d_{\mathfrak{q}}(m) / 2\right)$. Letting $L$ be the $m$-cover obtained by applying $\left(\mathrm{B}_{m}\right)$ with $S$ and $\mathfrak{q}$, we have $\operatorname{ind}_{\mathfrak{p}} \alpha^{M} \mid[L: M]_{\mathfrak{p}}$ for all $\mathfrak{p} \in S$. Hence, by (2-2), $L$ splits $\alpha$.

$\left(\mathrm{A}_{m}\right) \Longrightarrow\left(\mathrm{B}_{m}\right)$ : Let $S$ be any finite set of primes of $K$ and $\mathfrak{q} \in U_{2}$. For every $p \mid m$, if $\left|S \cap U_{p}\right| \geq 2$, let $S_{p}:=S$; otherwise form $S_{p}$ by adding to $S$ finite primes of $U_{p}$, so that $\left|S_{p} \cap U_{p}\right|=2$. Note that $\left(S_{2}, m\right)$ is unbalanceable if and only if $(S, m)$ is unbalanceable. If $\left(S_{2}, m\right)$ is balanceable, enlarge $S_{2}$ to assume that $\left(S_{2}, m\right)$ is balanced. 
For every $p \mid m$, construct $\alpha_{p}$ by applying Lemma 2.7 with $p, n=v_{p}(m), \mathfrak{q}$, and $S_{p}$. Set $\alpha=\sum_{p \mid m} \alpha_{p}$. Since $S_{p} \supseteq S$, by the definition of $d_{\mathfrak{p}}(m)$, the properties of $\alpha_{p}, p \mid m$, give ind $\alpha^{M}=m, \operatorname{ind}_{\mathfrak{p}} \alpha^{M}=\operatorname{gcd}(m, 2)$ for all real $\mathfrak{p} \in S$ which are unramified in $M$, and $\operatorname{ind}_{\mathfrak{p}} \alpha^{M}=d_{\mathfrak{p}}(m)$ for every finite $\mathfrak{p} \in S$, except for $\mathfrak{p}=\mathfrak{q}$ when $(S, m)$ is unbalanceable, in which case $\operatorname{ind}_{\mathfrak{q}} \alpha^{M}=d_{\mathfrak{q}}(m) / 2$. Applying $\left(\mathrm{A}_{m}\right)$ to $\alpha$, we obtain the desired cover $L$.

Remark 2.9. The proof of Proposition 2.8 shows that if there are noncrossed products $\alpha+\chi$ with ind $\alpha^{M}=m$, then there are infinitely many such noncrossed products. Indeed, if ( $\left.\mathrm{B}_{m}\right)$ fails for a set $S_{0}$, it fails for every set $S$ containing $S_{0}$, so that $(S, m)$ is balanceable if and only if $\left(S_{0}, m\right)$ is balanceable. The proof reveals that for every such set $S$, there is an $\alpha$ whose Hasse invariants are nonzero at primes of $S$ and $\alpha+\chi$ is a noncrossed product. In particular, there are infinitely many such classes $\alpha$.

Definition 2.10. For a prime $\mathfrak{p}$ of $K$, we say that the cover $L$ has full local degree at $\mathfrak{p}$ if $[L: M]_{\mathfrak{p}}=[L: M]$ for finite $\mathfrak{p}$, or if $[L: M]_{\mathfrak{p}}=\operatorname{gcd}(2,[L: M])$ for real $\mathfrak{p}$, or if $\mathfrak{p}$ is complex. For a set $S$ of primes of $K$, we say $L$ has full local degree in $S$ if $L$ has full local degree at each $\mathfrak{p} \in S$.

Note that $\left(\mathrm{B}_{m}\right)$ requires full local degree at every $\mathfrak{p} \in S \backslash U$.

Lemma 2.11. Let $m^{\prime} \mid m$. Suppose there is a finite nonempty set $S_{0}$ disjoint from $U$ such that any $m$-cover of $M / K$ with full local degree in $S_{0}$ contains an $m^{\prime}$-cover. Then $\left(\mathrm{B}_{m}\right)$ implies $\left(\mathrm{B}_{m^{\prime}}\right)$.

Proof. Let $\mathfrak{p}_{1}$ be a finite prime for which $v_{2}\left([M: K]_{\mathfrak{p}_{1}}\right)=u_{2}^{(1)}$. For a given finite set $S$, let $S^{\prime}:=S \cup S_{0}$. Note that there are three possible cases: (a) $\left(S^{\prime}, m^{\prime}\right)$ and $\left(S^{\prime}, m\right)$ are balanceable; (b) $\left(S^{\prime}, m^{\prime}\right)$ and $\left(S^{\prime}, m\right)$ are unbalanceable; (c) $\left(S^{\prime}, m^{\prime}\right)$ is balanceable and $\left(S^{\prime}, m\right)$ is not. Also note that (c) occurs only if $w_{2}\left(m^{\prime}, \mathfrak{p}_{1}\right)=0$.

If (b) occurs, fix a prime $\mathfrak{q} \in U_{2}$. Let $L$ be an $m$-cover obtained by applying $\left(\mathrm{B}_{m}\right)$ to $S^{\prime}, S^{\prime}$ and $\mathfrak{q}$, and $S^{\prime}$ and $\mathfrak{p}_{1}$ in cases (a), (b), (c), respectively. Since $S_{0} \cap U=\varnothing$, $L$ has full local degree in $S_{0}$. By assumption, $L$ contains an $m^{\prime}$-cover $L^{\prime}$ of $M / K$. Since $d_{\mathfrak{p}}(m) \mid[L: M]_{\mathfrak{p}}\left(\operatorname{resp} .\left(d_{\mathfrak{p}}(m) / 2\right) \mid[L: M]_{\mathfrak{p}}\right)$ implies $d_{\mathfrak{p}}\left(m^{\prime}\right) \mid\left[L^{\prime}: M\right]_{\mathfrak{p}}$ (resp. $\left.\left(d_{\mathfrak{p}}\left(m^{\prime}\right) / 2\right) \mid\left[L^{\prime}: M\right]_{\mathfrak{p}}\right)$ for all primes $\mathfrak{p}$ of $K, L^{\prime}$ satisfies $d_{\mathfrak{p}}\left(m^{\prime}\right) \mid\left[L^{\prime}: M\right]_{\mathfrak{p}}$ for all $\mathfrak{p} \in S$ except for $\mathfrak{p}=\mathfrak{q}$ when (b) holds, in which case $\left(d_{\mathfrak{q}}\left(m^{\prime}\right) / 2\right) \mid\left[L^{\prime}: M\right]_{\mathfrak{p}}$.

Reduction to prime powers. Having shown the equivalence of $\left(\mathrm{I}_{m}\right)$ and $\left(\mathrm{B}_{m}\right)$, we now consider $\left(\mathrm{B}_{m}\right)$. We provide choices of the set $S$ that enforce tight restrictions on the structure of $\operatorname{Gal}(L / K)$ for covers $L$ of $M / K$ with full local degree in $S$.

Our first usage of this strategy is in reducing $\left(\mathrm{B}_{m}\right)$ from arbitrary $m \in \mathbb{N}$ to prime powers. Except for the part concerning the characteristic of $K$ (Lemma 2.13 below), this reduction is identical to the corresponding one in [Hanke and Sonn 2011]. 
Let $m=\prod p^{n_{p}}$ be the prime factorization. By taking field composita of covers, if $\left(\mathrm{B}_{p^{n} p}\right)$ holds for all $p \mid m$, then $\left(\mathrm{B}_{m}\right)$ holds. We show:

Proposition 2.12. $\left(\mathrm{B}_{m}\right)$ holds if and only if $\left(\mathrm{B}_{p^{n} p}\right)$ holds for all $p \mid m$.

We first treat the wild case separately:

Lemma 2.13. If $p=$ char $K$ then $\left(\mathrm{B}_{p^{n}}\right)$ holds for all $n \in \mathbb{N}$.

Proof. Let $n \in \mathbb{N}$ and $S$ be a finite set of primes of $K$. For every $\mathfrak{p} \in S$ there is a cyclic $p^{n}$-extension $L^{\prime}(\mathfrak{p}) / K_{\mathfrak{p}}$ which is disjoint from $M_{\mathfrak{p}}$ (see [Koch 1970, Satz 10.4$\left.]^{6}\right)$. By the Grunwald-Wang theorem, there is a cyclic $p^{n}$-extension $L^{\prime} / K$ whose completion at $\mathfrak{p}$ is $L_{\mathfrak{p}}^{\prime}=L^{\prime}(\mathfrak{p})$ for all $\mathfrak{p} \in S$. Let $L:=L^{\prime} M$. Since $L_{\mathfrak{p}}^{\prime} \cap M_{\mathfrak{p}}=K_{\mathfrak{p}}$, one has $[L: M]_{\mathfrak{p}}=p^{n}$ for all $\mathfrak{p} \in S$. Thus, $L$ is a $p^{n}$-cover of $M / K$ with full local degree in $S$.

It remains to show that $\left(\mathrm{B}_{m}\right)$ implies $\left(\mathrm{B}_{p^{n} p}\right)$ for all $p \mid m$ with $p \neq \operatorname{char} K$.

Proof of Proposition 2.12. Let $p \mid m$ with $p \neq \operatorname{char} K$. By [Hanke and Sonn 2011, $\S 7$, p. 325 , Corollary], there are infinitely many primes $\mathfrak{p}$ of $K$ such that for any $m$-cover of $M / K$ with full local degree at $\mathfrak{p}$, the $p$-Sylow subgroup of $\operatorname{Gal}(L / M)$ has a complement $\operatorname{Gal}\left(L / M_{0}\right)$ which is normal in $\operatorname{Gal}(L / K)$, and hence $L$ contains a $p^{n}$-cover $M_{0}$ of $M / K$. (Note that the assumption $M / K$ cyclic is never used in the proof of [Hanke and Sonn 2011, §7, p. 325, Corollary].) Since $U$ is finite we can choose such $\mathfrak{p} \notin U$. The proof is completed by setting $S_{0}:=\{\mathfrak{p}\}$ in Lemma 2.11.

An invariant subgroup. We are now able to complete the proof of Theorem 1.1. As outlined in Section 2, and using Propositions 2.8 and 2.12, it remains to prove Proposition 2.14 below. Let $p \in \mathbb{P}$ be fixed.

Proposition 2.14. For any $n \in \mathbb{N},\left(\mathrm{B}_{p^{n}}\right)$ implies $\left(\mathrm{B}_{p^{n-1}}\right)$.

Indeed, the bound $b_{p}(\chi)$ of Theorem 1.1 is the maximal $n$ for which $\left(\mathrm{B}_{p^{n}}\right)$ holds if such a maximum exists, and $b_{p}(\chi)=\infty$ otherwise. For more details on the description of $b_{p}(\chi)$, see Corollary 2.17 below.

For any cover $L$ of $M / K$, we consider the group extension

$$
1 \rightarrow \operatorname{Gal}(L / M) \rightarrow \operatorname{Gal}(L / K) \rightarrow \operatorname{Gal}(M / K) \rightarrow 1 .
$$

We will analyze several kinds of constraints that are imposed on (2-9) by the condition that $L$ has full local degree in $S_{0}$, for certain chosen sets $S_{0}$. More precisely, after showing that the kernel $A:=\operatorname{Gal}(L / M)$ can be assumed to be abelian, we focus on constraints regarding the conjugation action of $B:=\mathrm{Gal}(M / K)$ on $A$. The analysis of this action is the main ingredient in the proofs of both Proposition 2.14 and Theorem 1.2 below.

\footnotetext{
${ }^{6}$ Koch's book has been translated into English. However, Theorem 10.4 in the English version contains a typo: "finitely generated" should be replaced by "on countably many generators".
} 
In view of Lemma 2.13, we assume from now on that $p \neq \operatorname{char} K$. Fix $p^{n}$ and set $T:=M \cap K\left(\mu_{p^{\infty}}\right)$. For the proof of Proposition 2.14, it will suffice to analyze the action of $\operatorname{Gal}(M / T)$ on $A$.

Lemma 2.15. There exists a finite set $S_{0}$ of primes of $K$ disjoint from $U$ such that for any $p^{n}$-cover $L$ of $M / K$ with full local degree in $S_{0}$, the kernel $A$ is abelian, the group $\operatorname{Gal}(M / T)$ acts trivially on $A$, and A has rank at most 2 .

Proof. At first fix a $\sigma \in \operatorname{Gal}(M / T)$. By the Chebotarev density theorem, the Galois extension $M\left(\mu_{p^{n}}\right) / K$ has infinitely many unramified finite primes $\mathfrak{P}$ of $M\left(\mu_{p^{n}}\right)$ whose Frobenius element restricts to the identity on $K\left(\mu_{p^{n}}\right)$ and to $\sigma$ on $M$. Of those infinitely many $\mathfrak{P}$, choose one such that $\mathfrak{p}:=\mathfrak{P} \cap K \notin U$ and $p$ does not divide the norm $N(\mathfrak{p})$. (There are only finitely many $\mathfrak{P}$ that do not satisfy this, since $p \neq$ char $K$.) Since $p \nmid N(\mathfrak{p})$ and $\mu_{p^{n}} \subset K_{\mathfrak{p}}$, we have $N(\mathfrak{p}) \equiv 1\left(\bmod p^{n}\right)$.

Assume a $p^{n}$-cover $L$ of $M / K$ has full local degree at the chosen $\mathfrak{p}$. Let $\mathfrak{P}$ be a prime of $L$ dividing $\mathfrak{p}$. The decomposition group $\operatorname{Gal}\left(L_{\mathfrak{P}} / K_{\mathfrak{p}}\right)$ then equals $\operatorname{Gal}\left(L / M^{\sigma}\right)$, where $M^{\sigma}$ is the fixed field of $\sigma$. We will show that $\operatorname{Gal}\left(L_{\mathfrak{P}} / K_{\mathfrak{p}}\right)$ is abelian, and thus $\sigma$ acts trivially on $\operatorname{Gal}(L / M)$ : since char $K \nmid N(\mathfrak{p}), L_{\mathfrak{P}} / K_{\mathfrak{p}}$ is tame. Therefore, $\operatorname{Gal}\left(L_{\mathfrak{P}} / K_{\mathfrak{p}}\right)$ is a metacyclic group, generated by the inertia group $I_{\mathfrak{P}}$ and the Frobenius element, with the Frobenius acting on $I_{\mathfrak{P}}$ by raising each element to the power $N(\mathfrak{p})$. Since $\mathfrak{p}$ is unramified in $M,\left|I_{\mathfrak{P}}\right|$ divides $p^{n}$. Hence $\operatorname{Gal}\left(L_{\mathfrak{P}} / K_{\mathfrak{p}}\right)$ is abelian, because $N(\mathfrak{p}) \equiv 1\left(\bmod p^{n}\right)$.

Now let $\Sigma$ be a set of generators of $\operatorname{Gal}(M / T)$, or $\Sigma=\{1\}$ if $M=T$. For each $\sigma \in \Sigma$, choose a prime $\mathfrak{p}_{\sigma}$ as described in the first paragraph of the proof. Then $S_{0}:=\left\{\mathfrak{p}_{\sigma} \mid \mathfrak{p} \in \Sigma\right\}$ has the desired property.

By Lemma 2.11, Proposition 2.14 is completed once we show:

Proposition 2.16. Let $S_{0}$ be as in Lemma 2.15. Any $p^{n}$-cover of $M / K$ with full local degree in $S_{0}$ contains a $p^{n-1}$-cover of $M / K$.

Proof. We have assumed $p \neq \operatorname{char} K$. Let $B=\operatorname{Gal}(M / K)$. Let $L$ be a $p^{n}$-cover of $M / K$ with full local degree in $S_{0}$. By Lemma 2.15, the kernel $A$ is abelian. The subgroup $A[p]$ of $p$-torsion elements is a characteristic subgroup and hence invariant under the action of $B$ (we say $B$-invariant). It suffices to find a $B$-invariant subgroup $A_{0} \leq A[p]$ of order $p$; then the fixed field $L^{A_{0}}$ is the desired $p^{n-1}$-cover.

If $A$ is cyclic then $A[p]$ itself is such a subgroup; hence, assume $A$ noncyclic for the rest of the proof.

Recall that $A[p]$ is an $\mathbb{F}_{p}$-vector space and that any action of some group $H$ on $A[p]$ is a representation of $H$ over $\mathbb{F}_{p}$. In this sense, the $H$-invariant subgroups of order $p$ are the $H$-invariant subspaces of dimension 1 .

By Lemma 2.15, $B$ acts on $A$ through $\operatorname{Gal}(T / K)$. Let $\operatorname{Gal}(T / K)=P \oplus C$ with $P$ the $p$-part and $|C|$ prime to $p$. Then $|C|$ divides $p-1$. Since $|C|$ is prime 
to $p$, any representation of $C$ over $\mathbb{F}_{p}$ is semisimple and hence decomposes into a product of irreducible representations. Since $\mathbb{F}_{p}$ contains the $|C|$-th roots of unity, the irreducible representations of $C$ are of dimension 1 . Thus, there is a $C$-invariant subgroup $A_{0} \leq A[p]$ of order $p$.

By Lemma 2.15, $A$ has rank 2, that is, $A[p] \cong C_{p} \times C_{p}$. This group has exactly $p+1$ order- $p$ subgroups, say $A_{0}, \ldots, A_{p}$, which are permuted by the action of $B$. Thus, we have an induced action of $B$ on the set of indices $\{0, \ldots, p\}$.

We know $A_{0}$ is $C$-invariant. If $A_{0}$ is $P$-invariant then $A_{0}$ is $B$-invariant and we are done. Assume $A_{0}$ is not $P$-invariant. Since $P$ is a $p$-group, there are two $P$-orbits on $\{0, \ldots, p\}$, say $\{0, \ldots, p-1\}$ and $\{p\}$. Since $P$ and $C$ commute and $A_{0}$ is $C$-invariant, each of $A_{0}, \ldots, A_{p-1}$ is also $C$-invariant. Hence the remaining subgroup $A_{p}$ is $C$-invariant and $P$-invariant. We found a $B$-invariant subgroup $A_{p}$ of $A$ with $\left|A_{p}\right|=p$. As noted in the beginning of the proof, this gives the assertion.

This completes the proof of Proposition 2.14, and hence of Theorem 1.1.

Summary. In addition to the existence of bounds (Theorem 1.1) we get:

Corollary 2.17. The bound $b_{p}(\chi)$ is the maximal $n$ such that for every finite set $S$ of primes of $K$ and $\mathfrak{q} \in U_{2}$, there is a $p^{n}$-cover $L$ of $M / K$ satisfying:

(i) $d_{\mathfrak{p}}\left(p^{n}\right) \mid[L: M]_{\mathfrak{p}}$ for all $\mathfrak{p} \in S$, except for $\mathfrak{p}=\mathfrak{q}$ when $p=2$ and $\left(S, 2^{n}\right)$ is unbalanceable, in which case $\left(d_{\mathfrak{q}}\left(2^{n}\right) / 2\right) \mid[L: M]_{\mathfrak{q}}$;

(ii) $A=\operatorname{Gal}(L / M)$ is abelian of rank at most 2;

(iii) $\operatorname{Gal}(M / T)$ acts trivially on A via conjugation in $\operatorname{Gal}(L / K)$.

If no maximal $n$ exists, then $b_{p}(\chi)=\infty$.

Proof. Let $S_{0}$ be a finite set of primes of $K$ that is disjoint from $U$. Suppose that any $p^{n}$-cover of $L$ with full local degree in $S_{0}$ has a certain property. Then this property can be added to the condition $\left(\mathrm{B}_{p^{n}}\right)$ without changing the truth value of $\left(\mathrm{B}_{p^{n}}\right)$, because the set $S$ in $\left(\mathrm{B}_{p^{n}}\right)$ can be enlarged by $S_{0}$. By Lemma 2.15, this argument applies to the properties (ii) and (iii). Hence, the corollary is a consequence of Proposition 2.14.

Remark 2.18. Regarding condition (i) in Corollary 2.17, if $\mathfrak{p}$ is not isolated, then $d_{\mathfrak{p}}\left(p^{n}\right) \mid[L: M]_{\mathfrak{p}}$ is equivalent to saying that $L$ has full local degree at $\mathfrak{p}$.

Regarding condition (iii) in Corollary 2.17, if $M$ and $K\left(\mu_{p^{\infty}}\right)$ are disjoint over $K$, then (iii) is equivalent to saying that the group extension (2-9) is central.

\section{Finiteness of bounds}

The exponents of kernels. Suppose we use the setup described in Section 2, so that $M / K$ is an abelian extension of global fields. Let $p \in \mathbb{P}$ be fixed and different 
from char $K$, and as before set $T:=K\left(\mu_{p^{\infty}}\right) \cap M$. In Section 2 we showed that suitable choices of the set $S$ put constraints on structure of covers $L$ of $M / K$ with full local degree in $S$, to the extent that the action of $\operatorname{Gal}(M / T)$ on $A=\operatorname{Gal}(L / M)$ is trivial. This was sufficient to prove the existence of the bounds. Now, in order to prove the finiteness of the bounds, we analyze constraints on the action of the entire group $B=\operatorname{Gal}(M / K)$ on $A$. The set $S$ for this purpose will be constructed from the families $Q_{\sigma}$, which we define next for each $\sigma \in B$.

Denote by $p^{s}$ the number of $p$-power roots of unity in $M$ and by $r$ the maximal number for which $\mu_{2^{r}} \subseteq M(\sqrt{-1})$. Let $U$ be the finite set defined in Definition 2.2, so that for every finite set $S$, condition $\left(\mathrm{B}_{m}\right)$ requires full local degree at every prime $\mathfrak{p} \in S \backslash U$.

Fix an element $\sigma \in B$ and let $f_{\sigma}$ be the order of the restriction $\sigma_{\mid T}$ of $\sigma$ to $T$. We define $Q_{\sigma}$ to be the set of all primes $\mathfrak{p} \notin U$ of $K$, unramified in $M$, whose Frobenius automorphism in $M / K$ is $\sigma$, and such that the norm $N(\mathfrak{p})$ is prime to $p$ and is of order strictly greater than $f_{\sigma}$ as an element of $\left(\mathbb{Z} / p^{s+1} \mathbb{Z}\right)^{*}(\operatorname{resp} . \bmod$ $\left(\mathbb{Z} / 2^{r+2} \mathbb{Z}\right)^{*}$ if $\left.p=2\right)$.

Lemma 3.1. For every $\sigma \in B$, the set $Q_{\sigma}$ is infinite.

Proof. Assume without loss of generality that $\sqrt{-1} \in M$. Otherwise, repeat the proof for a lift $\tau \in \operatorname{Gal}(M(\sqrt{-1}) / K)$ of $\sigma$ to deduce that $Q_{\tau}$ is infinite. Since $Q_{\tau} \subseteq Q_{\sigma}$ and $f_{\tau} \geq f_{\sigma}$, the assertion for $\sigma$ follows. Note that under this assumption we have $r=s$, so we will use only $s$ in the rest of the proof. Set $T^{\prime}:=K\left(\mu_{p^{s+1}}\right)$ (resp. $T^{\prime}:=K\left(\mu_{2^{s+2}}\right)$ if $\left.p=2\right)$ and note that $T^{\prime} \cap M=T$.

We first claim that $\operatorname{Gal}\left(T^{\prime} / K\right)$ contains an element $\sigma^{\prime}$ of order greater than $f_{\sigma}$ whose restriction to $T$ is $\sigma_{\mid T}$. For $s>0, T=K\left(\mu_{p^{s}}\right)$, and hence the group $\operatorname{Gal}\left(T / T^{\sigma}\right)$ is naturally identified with a subgroup $H$ of $\left(\mathbb{Z} / p^{s} \mathbb{Z}\right)^{*}$, and $\operatorname{Gal}\left(T^{\prime} / T^{\sigma}\right)$ is identified with the full preimage of $H$ under the natural projection

$$
\pi:\left(\mathbb{Z} / p^{s+1} \mathbb{Z}\right)^{*} \rightarrow\left(\mathbb{Z} / p^{s} \mathbb{Z}\right)^{*} \quad\left(\operatorname{resp} . \pi:\left(\mathbb{Z} / 2^{s+2} \mathbb{Z}\right)^{*} \rightarrow\left(\mathbb{Z} / 2^{s} \mathbb{Z}\right)^{*} \text { if } p=2\right) .
$$

The claim follows for $s>0$ since each element of $\left(\mathbb{Z} / p^{s} \mathbb{Z}\right)^{*}$ has a preimage under $\pi$ of a greater order. If $s=0$, then $p$ is odd, and the claim holds, as the restriction map $\operatorname{Gal}\left(T^{\prime} / T^{\sigma}\right) \rightarrow \operatorname{Gal}\left(T / T^{\sigma}\right)$ is an epimorphism of cyclic groups with nontrivial kernel.

Since $\sigma$ and $\sigma^{\prime}$ agree on $T$, Chebotarev's density theorem implies that there are infinitely many primes $\mathfrak{p}$ of $K$, with $p \nmid N(\mathfrak{p})$, whose Frobenius automorphism is $\sigma^{\prime}$ in $T^{\prime} / K$ and is $\sigma$ in $M / K$. Such primes are in $Q_{\sigma}$ since the order of the norm of $\mathfrak{p}$ as an element in $\left(\mathbb{Z} / p^{s+1} \mathbb{Z}\right)^{*}$ (resp. in $\left.\left(\mathbb{Z} / 2^{s+2} \mathbb{Z}\right)^{*}\right)$ is the same as the order of their Frobenius automorphism in $K\left(\mu_{p^{s+1}}\right) / K$ (resp. in $K\left(\mu_{2^{s+2}}\right) / K$ ).

For a prime $\mathfrak{p}$ of $K$, denote by $e_{\mathfrak{p}}(L / K)$ the ramification index of $\mathfrak{p}$ in a Galois extension $L / K$. 
Lemma 3.2. Let $S_{0}$ be as in Lemma 2.15 and let $\sigma \in B$. Suppose that a $p^{n}$-cover $L$ of $M / K$ has full local degree in $S_{0}$ and at $\mathfrak{p} \in Q_{\sigma}$. Then $e_{\mathfrak{p}}(L / K) \mid p^{s}$ if $p$ is odd and $e_{\mathfrak{p}}(L / K) \mid 2^{r+1}$ if $p=2$.

Proof. By Lemma 2.15, the kernel $A$ is abelian, $\operatorname{Gal}(M / T)$ acts trivially on $A$, and hence the action of $B$ on $A$ factors through the action of $\operatorname{Gal}(T / K)$. Thus, $\sigma$ acts on the inertia group $I \subseteq A$ of $\mathfrak{p}$ in $L / K$ as an automorphism of order at most $f_{\sigma}$.

Assume on the contrary that there is an element $a \in I$ of order $p^{s+1}$ (resp. $2^{r+2}$ if $p=2$ ). Since $\mathfrak{p}$ is tamely ramified in $L, \sigma$ acts on $I$ by raising each element to the power $N(\mathfrak{p})$ and hence defines an automorphism of order greater than $f_{\sigma}$ on $\langle a\rangle$, a contradiction.

We derive Theorem 1.2 from the following proposition, whose proof appears in the end of this subsection.

Proposition 3.3. Assume the p-Sylow subgroup of $B$ is noncyclic. Then there exists a finite set $S_{0}$ of primes of $K$ disjoint from $U$ such that for any $p^{n}$-cover $L$ of $M / K$ with full local degree in $S_{0}, \mathrm{Gal}(L / M)$ is abelian of rank at most 2 and exponent at most $p^{s}$ (resp. $2^{r+2}$ if $p=2$ ).

If the $p$-Sylow subgroup of $B$ is noncyclic, then Proposition 3.3 allows us to improve on Corollary 2.17 by adding the following property to the list:

(iv) $\exp A \mid p^{s}$ if $p$ is odd and $\exp A \mid 2^{r+2}$ if $p=2$.

In particular, since $A$ has rank at most $2, b_{p}(\chi) \leq 2 s$ if $p$ is odd and $b_{p}(\chi) \leq 2(r+2)$ if $p=2$. This proves Theorem 1.2.

The proof of Proposition 3.3 relies on the following group-theoretic proposition, whose proof is given starting on page 851 .

Proposition 3.4. Let

$$
1 \rightarrow A \rightarrow G \stackrel{\pi}{\rightarrow} B \rightarrow 1
$$

be an extension of nontrivial abelian p-groups $A, B$. If $B$ is noncyclic and $\pi^{-1}\langle x\rangle$ is cyclic for all $x \in B$, then $|A|=2$.

Proof of Proposition 3.3. Replacing $K$ with the fixed field of the $p$-Sylow subgroup of $B$, we can assume without loss of generality that $B$ is a $p$-group. By the assumptions of the proposition, $B$ is a noncyclic abelian group.

Choose $S_{0}$ to be the set from Lemma 2.15 joined with one $\mathfrak{p}_{\sigma} \in Q_{\sigma}$ for each $\sigma \in B$. Suppose $L$ is a $p^{n}$-cover of $M / K$ with full local degree in $S_{0}$.

By Lemma 2.15, $A$ is abelian of rank at most 2. Since the $p^{s}$-torsion subgroup $A\left[p^{S}\right]$ is a characteristic subgroup of $A$, it is a normal subgroup of $\operatorname{Gal}(L / K)$ and hence the fixed field $L_{0}:=L^{A\left[p^{s}\right]}$ is Galois over $K$. If $p=2$, then we consider $L_{0}:=L^{A\left[2^{r+1}\right]}$ instead, which is also Galois over $K$. To prove our claim it suffices to show that $L_{0}=M\left(\operatorname{resp} .\left[L_{0}: M\right] \leq 2\right.$ if $\left.p=2\right)$. 
Fix an element $\sigma \in B$ and let $\mathfrak{P}_{\sigma}$ be a prime of $L$ which divides $\mathfrak{p}_{\sigma}$. Let $I_{\sigma} \subseteq A$ be the inertia group of $\mathfrak{P}_{\sigma}$ in $L / K$. By Lemma 3.2, $\left|I_{\sigma}\right| \leq p^{s}$ (resp. $\left|I_{\sigma}\right| \leq 2^{r+1}$ ) and hence $I_{\sigma} \subseteq A\left[p^{s}\right]$ (resp. $I_{\sigma} \subseteq A\left[2^{r+1}\right]$ ). Thus, $L_{0} / K$ is unramified at $\mathfrak{p}_{\sigma}$ and hence $\mathfrak{P}_{\sigma} \cap L_{0}$ has a cyclic decomposition group in $L_{0} / K$. Since $L_{0}$ has full local degree at $\mathfrak{p}$, the decomposition group of $\mathfrak{P}_{\sigma} \cap L_{0}$ in $L_{0} / K$ is $\operatorname{Gal}\left(L_{0} / M^{\sigma}\right)$, and hence $L_{0} / M^{\sigma}$ is a cyclic extension.

Since $L_{0} / M^{\sigma}$ is cyclic for all $\sigma \in B$ and since $B$ is noncyclic, Proposition 3.4 applied to the group extension

$$
1 \rightarrow \operatorname{Gal}\left(L_{0} / M\right) \rightarrow \operatorname{Gal}\left(L_{0} / K\right) \rightarrow \operatorname{Gal}(M / K) \rightarrow 1
$$

shows $L_{0}=M\left(\operatorname{resp} .\left[L_{0}: M\right] \leq 2\right.$ if $\left.p=2\right)$, proving the claim.

Central group extensions. The last ingredient is a proof of Proposition 3.4. We begin with elementary properties of commutators in a central group extension

$$
1 \rightarrow A \rightarrow G \rightarrow B \rightarrow 1
$$

of abelian groups $A, B$. Let $s: B \rightarrow G$ be a section of $G \rightarrow B$ (not necessarily a homomorphism).

Lemma 3.5. Commutators in $G$ are bimultiplicative. That is, the map

$$
\beta: B \times B \rightarrow A, \quad(x, y) \mapsto[s(x), s(y)]
$$

does not depend on the choice of $s$ and is bimultiplicative.

Proof. Since $[G, G] \subseteq A \subseteq Z(G)$, we have

$$
[a b, x]=a b x b^{-1} a^{-1} x^{-1}=a\left(b x b^{-1} x^{-1}\right) x a^{-1} x^{-1}=[a, x][b, x] .
$$

Similarly one checks $[x, a b]=[x, a][x, b]$, that is, that commutators are bimultiplicative. The statement about $\beta$ follows from this.

We next look at the meaning of the condition that $\pi^{-1}\langle x\rangle$ is cyclic for $x \in B$. For $x=1$ it means $A$ is cyclic, and for $x \neq 1$ one has:

Lemma 3.6. Assume $A$ is cyclic. For $x \in B, x \neq 1, \pi^{-1}\langle x\rangle$ is cyclic if and only if $A$ is trivial or generated by $s(x)^{\text {ord } x}$.

In order to prove Proposition 3.4, we now assume $A, B$ are nontrivial $p$-groups and $A$ is cyclic. The map

$$
\gamma: B[p] \rightarrow A / A^{p}, \quad x \mapsto s(x)^{p}
$$

is obviously independent of the choice of $s$.

Lemma 3.7. Assume A, B are nontrivial p-groups and $A$ is cyclic.

(i) For $x \in B[p], x \neq 1, \pi^{-1}\langle x\rangle$ is cyclic if and only if $\gamma(x) \neq 1$. 
(ii) If $p$ is odd then $\gamma$ is a homomorphism.

(iii) If $p=2$ then $\gamma$ is a homomorphism if and only if $\beta(x, y) \in A^{2}$ for all $x, y$ in $B[2]$.

Proof. (i) is Lemma 3.6. Since $[G, G] \subseteq A \subseteq Z(G)$, we have $(s(x) s(y))^{p}=$ $s(x)^{p} s(y)^{p} \beta(x, y)^{p(p-1) / 2}$ for all $x, y \in B$. In particular, for all $x, y \in B[p]$,

$$
\gamma(x y)=s(x y)^{p} A^{p}=(s(x) s(y))^{p} A^{p}=\gamma(x) \gamma(y) \beta(x, y)^{p(p-1) / 2} .
$$

Thus $\gamma$ is a homomorphism if and only if $\beta(x, y)^{p(p-1) / 2} \in A^{p}$. If $p=2$ then $p(p-1) / 2=1$, proving (iii). For $p$ odd, we use Lemma 3.5 to see that $\beta(x, y) \in$ $A[p]$ for all $x, y \in B[p]$, so the term $\beta(x, y)^{p(p-1) / 2}$ vanishes.

Proof of Proposition 3.4. Let $A, B$ be nontrivial abelian $p$-groups, $A$ cyclic and $B$ noncyclic. By hypothesis and Lemma 3.7(i), $\gamma(x) \neq 1$ for all $x \in B[p], x \neq 1$. Therefore, if $\gamma$ is a homomorphism then it is injective, in contradiction to $A$ being cyclic and $B$ noncyclic. Hence, $\gamma$ is not a homomorphism. By parts (ii) and (iii) of Lemma 3.7, we have $p=2$ and an element $a:=\beta(x, y) \notin A^{2}$ for some $x, y \in B$ [2]. By Lemma 3.5, $a \in A[2] \backslash A^{2}$ and hence $|A|=2$.

\section{Examples}

Suppose we are in the setup described in Section 2. In particular, $\alpha \in \operatorname{Br}(K)$, $\chi \in \operatorname{Hom}\left(G_{K}, \Delta / \Gamma\right)$, and $M$ is the fixed field of ker $\chi$, an abelian extension of $K$. In this section we provide examples of noncrossed products with the smallest possible indices in fibers over noncyclic $\chi$.

For $p \in \mathbb{P}$, let $p^{s_{p}(M)}$ denote the number of $p$-power roots of unity in $M$. If $\alpha+\chi$ has index equal to $|\chi|$, then the division algebra contained in $\alpha+\chi$ is a crossed product, because $\alpha$ is split by $M$. Therefore, noncrossed products of index $p^{2}$ are possible only if $|\chi|=p$, in particular only if $\chi$ is cyclic.

Suppose $\chi$ is noncyclic with $|\chi|=p^{2}$. If $b_{p}(\chi)=0$, then the fiber over $\chi$ contains infinitely many noncrossed products of index $p|\chi|$. By Theorem 1.2, this happens, for example, whenever $s_{p}(M)=0$. We give examples of bicyclic $\chi$ with $|\chi|=p^{2}$ and $b_{p}(\chi)=0$ but $s_{p}(M) \geq 1$. Note that such a phenomenon is in contrast to the case of cyclic $\chi$, for which one always has $b_{p}(\chi) \geq s_{p}(M)$ (see [Hanke and Sonn 2011]).

For $p=2$, an example as described above was given over $K=\mathbb{Q}$ in [Hanke 2004 ] and over $K=\mathbb{F}_{q}(t)$ for all $q \equiv 3(\bmod 8)$ in [Coyette 2012, Example 2.8]. These turn out to be special cases of our Examples 4.1 and 4.3 below.

We start with $K=\mathbb{Q}$ and $p=2$ :

Example 4.1. Let $q, \ell$ be odd primes such that $q \equiv 3(\bmod 4), q \not \equiv-\ell(\bmod 8)$, and $q$ is a nonsquare modulo $\ell$. Note that for any odd prime $\ell$, a suitable $q$ can be 
chosen using Dirichlet's theorem. ${ }^{7}$ Let $M:=\mathbb{Q}(\sqrt{q}, \sqrt{-\ell})$. Corollary 2.17 applied with $S=\{\ell\}$ and the following claim show $b_{p}(\chi)=0$.

Claim. The extension $M / \mathbb{Q}$ has no isolated primes and there is no 2-cover $L$ of $M / K$ with local degree $[L: M]_{\ell}=2$.

Proof. Set $K_{1}:=\mathbb{Q}(\sqrt{q}), K_{2}:=\mathbb{Q}(\sqrt{-\ell})$ and let $\chi$ be a character for which the fixed field of $\operatorname{ker} \chi$ is $M$.

We first check that $M / \mathbb{Q}$ has no isolated primes. The prime $\ell$ ramifies in $K_{2}$ and is inert in $K_{1}$, so $\left[M: \mathbb{Q}_{\ell}=4\right.$.

Case $\ell \equiv 3(\bmod 4)$ : By reciprocity, $\ell$ is a square modulo $q$, and hence $-\ell$ is a nonsquare modulo $q$. The prime $q$ thus ramifies in $K_{1}$ and is inert in $K_{2}$, so $[M: \mathbb{Q}]_{q}=4$.

Case $\ell \equiv 1(\bmod 4):$ Since $q \not \equiv-\ell(\bmod 8)$, we have $\mathbb{Q}_{2}(\sqrt{q}) \neq \mathbb{Q}_{2}(\sqrt{-\ell})$, so $[M: \mathbb{Q}]_{2}=4$. In any case, $M / \mathbb{Q}$ has no isolated prime.

Now assume $L$ is a 2-cover of $M / \mathbb{Q}$ with full local degree at $\ell$. Since $K_{1}$ is real and $M$ is not, $M / K_{1}$ does not have a cyclic 2 -cover. Since $q \equiv 3(\bmod 4),-1$ is not a square in $\mathbb{Q}_{q}$. This implies that $\mathbb{Q}_{q}$ does not have any totally ramified degree-4 extension, so that any ramified quadratic extension of $\mathbb{Q}_{q}$ cannot have a cyclic 2 -cover. Thus, globally, $K_{1} / \mathbb{Q}$ does not have a cyclic 2 -cover. The inertia field of $\ell$ in $L / \mathbb{Q}$ contains $K_{1}$ and is cyclic over $\mathbb{Q}$, and thus is equal to $K_{1}$. This is a contradiction because $L$ is then a cyclic 2-cover of $M / K_{1}$.

Remark. (i) Suppose $M / \mathbb{Q}$ is as in Example 4.1. Consider $\alpha \in \operatorname{Br}(\mathbb{Q})$ such that ind $\alpha=8$ and ind $\alpha^{\mathbb{Q}(\chi)}=2$. Since $\ell$ is not 2-isolated in $M / \mathbb{Q}$, we can find such an $\alpha$ with $\operatorname{ind}_{\ell} \alpha=8$. Since $M / \mathbb{Q}$ does not have a 2-cover $L$ with $[L: M]_{\ell}=2$, no 2-cover of $M / \mathbb{Q}$ splits $\alpha$. Hence, the underlying division algebra of $\alpha+\chi$ is a noncrossed product of index 8 .

(ii) In Example 4.1 we can choose $(\ell, q)=(3,11),(5,7),(7,3)$, etc. The example in [Hanke 2004] is the case $\ell=7$ and $q=3$.

We now turn to arbitrary global fields $K$ and a prime $p \neq$ char $K$. Example 4.1 does not generalize immediately because its proof uses a real prime. The following argument uses a third finite prime instead of a real prime:

Proposition 4.2. Let $K$ be a global field and let $p \in \mathbb{P}$ with $p \neq$ char $K$. Assume $s:=s_{p}(K)>0$. Let $\mathfrak{p}$ be any prime of $K$ with $p \nmid N(\mathfrak{p})$. There exists a bicyclic extension $M / K$ with group $C_{p^{s}} \times C_{p^{s}}$ and without isolated primes such that no p-cover $L$ of $M / K$ has $[L: M]_{\mathfrak{p}}=p$.

\footnotetext{
${ }^{7}$ Using the reciprocity law, it is also possible to choose a suitable $\ell$ for any prime $q \equiv 3(\bmod 4)$.
} 
Proof. By Chebotarev's density theorem, there are primes $\mathfrak{q}_{1}, \mathfrak{q}_{2}$ of $K$ such that $N\left(\mathfrak{q}_{i}\right) \equiv 1\left(\bmod p^{s}\right)$ but $N\left(\mathfrak{q}_{i}\right) \not \equiv 1\left(\bmod p^{s+1}\right)$. By the Grunwald-Wang theorem, there are cyclic extensions $K_{i} / K$ of degree $\left[K_{i}: K\right]=p^{s}$ such that in $K_{1}, \mathfrak{p}$ is inert, $\mathfrak{q}_{1}$ is totally ramified, and $\mathfrak{q}_{2}$ splits completely; and in $K_{2}, \mathfrak{q}_{1}$ is inert and $\mathfrak{p}$ and $\mathfrak{q}_{2}$ are totally ramified. Since $\mathfrak{p}$ and $\mathfrak{q}_{1}$ both have full local degree in $M, M / K$ has no isolated primes.

Since $N\left(\mathfrak{q}_{1}\right) \not \equiv 1\left(\bmod p^{s+1}\right)$ and $\mathfrak{q}_{1}$ is totally ramified in $K_{1} / K, K_{1} / K$ does not have a cyclic $p$-cover. Similarly, considering $\mathfrak{q}_{2}, M / K_{1}$ does not have a cyclic p-cover.

Assume on the contrary there is a $p$-cover $L$ of $M / K$ with $[L: M]_{\mathfrak{p}}=p$. Since the inertia field of $\mathfrak{p}$ contains $K_{1}$ and is cyclic over $K$, it equals $K_{1}$. This shows that $L$ is a cyclic $p$-cover of $M / K_{1}$, a contradiction.

Example 4.3. Let $p \in \mathbb{P}$ and $K=\mathbb{F}_{q}(t)$ for $q \equiv 1(\bmod p)$, so that $s:=s_{p}(K)>0$. Assume $a \notin\left(K^{\times}\right)^{p}$. Let $M=K\left(\sqrt[p^{s}]{t}, \sqrt[p^{s}]{(t-1)(t-a)}\right)$. By the following claim, the proof of Proposition 4.2 applies to $M$ and the primes $\mathfrak{p}=(t-a), \mathfrak{q}_{1}=(t), \mathfrak{q}_{2}=$ $(t-1)$. Therefore, $b_{p}(\chi)=0$ for any $\chi$ for which $M$ is the fixed field of $\operatorname{ker} \chi$.

For $q \equiv 3(\bmod 4)$ and $p=2$, Example 4.3 is identical to [Coyette 2012, Example 2.8].

Claim. Let $K_{1}=K\left(\sqrt[p^{s}]{t}\right), K_{2}=K\left(\sqrt[p^{s}]{(t-1)(t-a)}\right)$. Then $(t-a)$ is inert in $K_{1}$ and totally ramified in $K_{2},(t)$ is totally ramified in $K_{1}$ and inert in $K_{2}$, and $(t-1)$ splits completely in $K_{1}$ and is totally ramified in $K_{2}$.

Proof. In $K_{1}$ we have: $t \equiv a(\bmod t-a)$ is not a $p$-th power and $t \equiv 1(\bmod t-1)$ is a $p^{s}$-th power, and hence $(t-a)$ is inert, $(t-1)$ splits completely, and $(t)$ is totally ramified.

In $K_{2}$ we have: $(t-1)(t-a) \equiv a(\bmod t)$ is not a $p$-th power, and hence $(t)$ is inert and $(t-1),(t-a)$ are totally ramified.

\section{Acknowledgments}

The authors would like to thank Eli Aljadeff for the financial support of Hanke's visit to the Technion in 2010 and Julia Hartmann for supporting the visit of Neftin to RWTH Aachen in 2009. We also thank the referee for very helpful remarks which improved this paper. This material is based upon work supported by the National Science Foundation under Award No. DMS-1303990.

\section{References}

[Aljadeff et al. 2007] E. Aljadeff, J. Sonn, and A. R. Wadsworth, "Projective Schur groups of Henselian fields", J. Pure Appl. Algebra 208:3 (2007), 833-851. MR 2007k:11196 Zbl 1113.11066 [Amitsur 1972] S. A. Amitsur, “On central division algebras”, Israel J. Math. 12 (1972), 408-420. MR 47 \#6763 Zbl 0248.16006 
[Brussel 1995] E. Brussel, "Noncrossed products and nonabelian crossed products over $\mathbb{Q}(t)$ and $\mathbb{Q}((t))$ ", Amer. J. Math. 117:2 (1995), 377-393. MR 96a:16014 Zbl 0822.16013

[Brussel 2001] E. S. Brussel, "Noncrossed products over $k_{\mathfrak{p}}(t)$ ", Trans. Amer. Math. Soc. 353:5 (2001), 2115-2129. MR 2001j:16022 Zbl 0971.16009

[Brussel 2002] E. S. Brussel, "Non-crossed products over function fields", Manuscripta Math. 107:3 (2002), 343-353. MR 2003f:16027 Zbl 1009.16019

[Chen 2010] F. Chen, "Indecomposable and noncrossed product division algebras over curves over complete discrete valuation rings", 2010. arXiv 1004.3935

[Coyette 2012] C. Coyette, "Mal'cev-Neumann rings and noncrossed product division algebras", $J$. Algebra Appl. 11:3 (2012), 1250052, 12. MR 2928120 Zbl 1263.16022

[Hanke 2004] T. Hanke, "An explicit example of a noncrossed product division algebra", Math. Nachr. 271 (2004), 51-68. MR 2005e:16044 Zbl 1063.16025

[Hanke 2011] T. Hanke, "Galois subfields of inertially split division algebras", J. Algebra 346 (2011), 147-151. MR 2842074 Zbl 1248.16017

[Hanke and Sonn 2011] T. Hanke and J. Sonn, "The location of noncrossed products in Brauer groups of Laurent series fields over global fields", Math. Ann. 350:2 (2011), 313-337. MR 2012i:16037 Zbl 1242.16021

[Jacob and Wadsworth 1990] B. Jacob and A. Wadsworth, "Division algebras over Henselian fields", J. Algebra 128:1 (1990), 126-179. MR 91d:12006 Zbl 0692.16011

[Koch 1970] H. Koch, Galoissche Theorie der p-Erweiterungen, Springer, 1970. MR 45 \#233 Zbl 0216.04704

[Pierce 1982] R. S. Pierce, Associative algebras, Graduate Texts in Mathematics 88, Springer, New York, 1982. MR 84c:16001 Zbl 0497.16001

[Scharlau 1969] W. Scharlau, "Über die Brauer-Gruppe eines Hensel-Körpers", Abh. Math. Sem. Univ. Hamburg 33 (1969), 243-249. MR 41 \#185 Zbl 0179.07804

[Tignol and Wadsworth $\geq 2014$ ] J.-P. Tignol and A. Wadsworth, "Value functions on simple algebras, and associated graded algebras", In preparation.

[Witt 1936] E. Witt, “Schiefkörper über diskret bewerteten Körpern”, J. Reine Angew. Math. 176 (1936), 153-156. Zbl 0016.05102

Communicated by Raman Parimala

Received 2012-11-01 Revised 2013-10-28 Accepted 2013-12-05

hanke@math.rwth-aachen.de Lehrstuhl D für Mathematik, Rheinisch-Wesfälische Technische Hochschule Aachen, Templergraben 64, D-52062 Aachen, Germany

neftin@umich.edu

Department of Mathematics, University of Michigan, 530 Church St., Ann Arbor, MI 48109-1043, United States

sonn@math.technion.ac.il

Department of Mathematics, Technion-Israel Institute of Technology, Technion City, 32000 Haifa, Israel 


\section{Algebra \& Number Theory}

msp.org/ant

\section{EDITORS}

MANAGING EDITOR

Bjorn Poonen

Massachusetts Institute of Technology

Cambridge, USA

\author{
EDITORIAL BOARD CHAIR \\ David Eisenbud \\ University of California \\ Berkeley, USA
}

BOARD OF EDITORS

Georgia Benkart

Dave Benson

Richard E. Borcherds

John H. Coates

J-L. Colliot-Thélène

Brian D. Conrad

Hélène Esnault

Hubert Flenner

Edward Frenkel

Andrew Granville

Joseph Gubeladze

Roger Heath-Brown

Craig Huneke

Yujiro Kawamata

János Kollár

Yuri Manin

Barry Mazur

Philippe Michel

Susan Montgomery
University of Wisconsin, Madison, USA

University of Aberdeen, Scotland

University of California, Berkeley, USA

University of Cambridge, UK

CNRS, Université Paris-Sud, France

University of Michigan, USA

Freie Universität Berlin, Germany

Ruhr-Universität, Germany

University of California, Berkeley, USA

Université de Montréal, Canada

San Francisco State University, USA

Oxford University, UK

University of Virginia, USA

University of Tokyo, Japan

Princeton University, USA

Northwestern University, USA

Harvard University, USA

École Polytechnique Fédérale de Lausanne

University of Southern California, USA
Shigefumi Mori

Raman Parimala

Jonathan Pila

Anand Pillay

Victor Reiner

Peter Sarnak

Joseph H. Silverman

Michael Singer

Vasudevan Srinivas

J. Toby Stafford

Bernd Sturmfels

Richard Taylor

Ravi Vakil

Michel van den Bergh

Marie-France Vignéras

Kei-Ichi Watanabe

Efim Zelmanov

Shou-Wu Zhang
RIMS, Kyoto University, Japan

Emory University, USA

University of Oxford, UK

University of Notre Dame, USA

University of Minnesota, USA

Princeton University, USA

Brown University, USA

North Carolina State University, USA

Tata Inst. of Fund. Research, India

University of Michigan, USA

University of California, Berkeley, USA

Harvard University, USA

Stanford University, USA

Hasselt University, Belgium

Université Paris VII, France

Nihon University, Japan

University of California, San Diego, USA

Princeton University, USA

\section{PRODUCTION}

production@msp.org

Silvio Levy, Scientific Editor

See inside back cover or msp.org/ant for submission instructions.

The subscription price for 2014 is US $\$ 225 /$ year for the electronic version, and $\$ 400 /$ year ( $\$ 55$, if shipping outside the US) for print and electronic. Subscriptions, requests for back issues and changes of subscribers address should be sent to MSP.

Algebra \& Number Theory (ISSN 1944-7833 electronic, 1937-0652 printed) at Mathematical Sciences Publishers, 798 Evans Hall \#3840, c/o University of California, Berkeley, CA 94720-3840 is published continuously online. Periodical rate postage paid at Berkeley, CA 94704, and additional mailing offices.

ANT peer review and production are managed by EditFLOw ${ }^{\circledR}$ from Mathematical Sciences Publishers.

\section{PUBLISHED BY}

mathematical sciences publishers

nonprofit scientific publishing

http://msp.org/

(C) 2014 Mathematical Sciences Publishers 


\section{Algebra \& Number Theory}

Volume $8 \quad$ No. $4 \quad 2014$

The derived moduli space of stable sheaves

Kai Behrend, Ionut Ciocan-Fontanine, Junho Hwang and Michael Rose

Averages of the number of points on elliptic curves

Greg Martin, Paul Pollack and Ethan Smith

Noncrossed product bounds over Henselian fields

Timo Hanke, DanNy NeFtin and JaCk SONN

Yangians and quantizations of slices in the affine Grassmannian

Joel Kamnitzer, Ben Webster, Alex Weekes and Oded Yacobi

Equidistribution of values of linear forms on quadratic surfaces

OLIVER SARGENT

Posets, tensor products and Schur positivity

Vyjayanthi Chari, Ghislain Fourier and Daisuke SAGaKi

Parameterizing tropical curves I: Curves of genus zero and one

DAVID E. SPEYER

Pair correlation of angles between reciprocal geodesics on the modular surface

Florin P. Boca, Vicențiu Pașol, AleXandru A. Popa and AleXandru

ZAHARESCU

Étale contractible varieties in positive characteristic

ARMin HolschbaCh, JohanNes SCHMIDT and JaKob STIX 